\title{
Stem Cell Therapy for Myocardial Infarction: Challenges and Prospects
}

Gizaw Dabessa Satessa ${ }^{1 *}$, Jima Likisa Lenjisa ${ }^{3}$, Esayes Taddese Gebremariam² and Minyahil Alebachew Woldu ${ }^{3}$

${ }^{1}$ Department of Pharmacology, College of Medicine and Health Sciences, Ambo University, Ethiopia

${ }^{2}$ Department of Pharmacoepidemiology and Social Pharmacy, College of Medicine and Health Sciences, Ambo University, Ethiopia

${ }^{3}$ Department of Clinical Pharmacy, College of Medicine and Health Sciences, Ambo University, Ethiopia

\begin{abstract}
Myocardial infarction causes death worldwide with the greatest incidence being in the United States. Although there have been many advances in myocardial re-perfusion strategies and novel pharmacological approaches, therapies for treating acute and chronic myocardial ischemic damage remain limited. This means that no currently available heart failure treatment has demonstrated an ability to generate new muscle tissue within the scared regions of the heart. Stem cell, however, offers new hope to patients who have otherwise limited choices. Therefore, this review aims at exploring the use and peculiarities of stem cell therapy for myocardial infarction. But the success of stem cell therapy for clinical use needs the validation of several issues ranging from selection of appropriate stem cells, routes of transfer, establishment of conducive trans-differentiation milieu with associated cytokines, means to evaluate/track response to cell therapy to compliance with regulatory and ethical issues besides addressing biological and technical issues surrounding stem cell therapy.
\end{abstract}

Keywords: Heart; Myocardial infarction; Stem cell; Therapy

Abbreviations: ACC: American College of Medicine; ACE: Angiotensin Converting Enzyme; ADSCs: Adipose Derived Stem Cells; AHA: American Health Association; AMI: Acute Myocardial Infarction; BMGPs: Bone Morphogenic Proteins; BMSCs: Bone Marrow Derived Stem Cells; CD: Cluster of Differentiation; EPC: Endothelial Progenitor Cell; FGFs: Fibroblast Growth Factors; HSCs: Hematopoietic Stem Cells; ICD: Implantable Cardioverter-Defibrilator; LV: Left Ventricle; LVEF: Left Ventricle Ejection Fraction; MI: Myocardial Infarction; MRI: Magnetic Resonance Imaging; MSCs: Mesenchymal Stem Cells; PET: Positron Emission Tomography; SDF1: Stromal Cell Derived Factor 1; SPECT: Single Photon Emission Computed Tomography; VEGF: Vascular Endothelial Growth Factors

\section{Introduction}

Myocardial infarction (MI) is a main cause of mortality and morbidity in Western societies [1]. In USA the estimated annual incidence of MI is 610000 new attacks and 325000 recurrent attacks [2]. The American Heart Association (AHA) recently created a new set of 'Impact Goals' for the current decade. The aim, by 2020, is to improve the cardiovascular health of all Americans by $20 \%$, while reducing deaths from cardiovascular disease and stroke by $20 \%$ [3].

Although there have been many advances in myocardial reperfusion strategies and novel pharmacological approaches, therapies for treating acute and chronic myocardial ischemic damage remain limited. That means, no currently available heart failure treatment has demonstrated an ability to generate new muscle tissue within the scarred regions of a heart. Stem cell therapy, however, offers new hope to patients who have otherwise limited choices [4].

In recent years, the understanding that regenerative processes exist at the level of the myocardium has placed stem cell research at center stage in cardiology. Through cellular therapies, the concept of "growing" heart muscle and vascular tissue and manipulating the myocardial cellular environment has revolutionized the approach to treating heart disease. Unfortunately, however, the vast field of possibilities opened by stem cell therapy has frequently given rise to more questions than answers [5]. Therefore, this review aims at exploring the peculiarities of stem cell therapy for MI besides outlining the limitations of stem cell therapy for MI with respect to ethical, biological and technical issues.

\section{Rationale for Stem Cell Therapeutic Approaches Attributes of stem cells}

Stem cells are unspecialized cells that have two defining properties; the ability to self-regenerate and the ability to differentiate into other cell types. The biological principle that underlies stem cell therapy is tissue-directed differentiation. For example, adult stem cells isolated from liver tissue and re-injected into liver become hepatocytes, whereas the same cells injected into myocardium become myocytes. Furthermore, stem cells have been engrafted in to a broad spectrum of tissues, including regenerating bone, neural tissue, dystrophic skeletal muscle, and injured skeletal muscle [6].

\section{Integration of stem cells with viable myocardium}

Normal embryologic development of the myocardium (not to mention the whole heart, with its valve and complex architecture) depends on a series of coordinated, sequential, irreversible events (cellular differentiation, genetic expression, migration, and autocrine secretion) that are hard to imagine as being reproducible in the adult heart under normal conditions. Although transplanting stem cells will indeed develop into myocardial cells, this approach can hardly solve the problem of effective myocardial regrowth throughout the entire infracted area. Stem cell migration into an environment so different from the embryonic cardiac jelly seems quite unlikely; at the same time, satisfactory spatial and functional integration of the new myocytes into the remnant of the viable myocardium would likely constitute a formidable challenge [7].

*Corresponding author: Gizaw Dabessa Satessa, Department of Pharmacology, College of Medicine and Health Sciences, Ambo University, P.O. Box: 19, Ambo, Ethiopia, Tel: +251917305585; E-mail: gizawdhabessa@gmail.com

Received September 17, 2014; Accepted March 11, 2015; Published March 13, 2015

Citation: Satessa GD, Lenjisa JL, Gebremariam ET, Woldu MA (2015) Stem Cell Therapy for Myocardial Infarction: Challenges and Prospects. J Stem Cell Res Ther 5: 270. doi:10.4172/2157-7633.1000270

Copyright: (c) 2015 Satessa GD, et al. This is an open-access article distributed under the terms of the Creative Commons Attribution License, which permits unrestricted use, distribution, and reproduction in any medium, provided the original author and source are credited. 
Recent observations in the adult heart have suggested that adult cardiac and non-cardiac stem cells, such as those obtained from the bone marrow, brain, skeletal muscle, adipose tissue, liver, or peripheral blood may become cardiomyocytes after undergoing natural migration or experimental transplantation into the heart. This evidence indicates that the presence of such cells in the adult extracellular cardiac environment (which may need to be ischemic or damaged for this phenomenon to occur) induces the maturation of cardiac phenotypes [7].

The pivotal finding by Ashahara and colleagues that postnatal vasculogenesis exists (i.e. that stem cells contribute directly to the formation of new blood vessels in adults) provided new insights into mechanisms of cardiac repair. In the adult, revascularization does not rely exclusively on angiogenesis (sprouting from preexisting blood vessels). Furthermore, endothelial progenitor cells (EPCs) that originate in the bone marrow play a role in vasculogenesis (physiological and pathological) and circulate in adult peripheral blood. The intriguing observation in heart transplant patients that putative stem cells and progenitor cells from a recipient were present in the transplanted heart further supports the notion of ongoing regenerative and reparative mechanism mediated by circulating stem cells from the bone marrow [5].

\section{Sources of stem cells for cardiac repair}

Over the past decade, several indications using a variety of different types of stem cells have emerged including embryonic stem, adipose derived stem cells, peripheral blood stem cells and skeletal myoblasts for use in acute MI, chronic ischemia, and chronic heart failure. In addition to cellular therapy for the existing heart, some groups are attempting to create a bioartificial heart using cardiac or endothelial cells [4].

The recent discovery of cardiac stem cells obtained directly from adult cardiac tissue could lead to new treatments for heart failure patients [8]. It is believed that these cells are inherently programmed to reconstitute cardiac tissue and play a role in the clinical benefit observed in other stem cell trials. The proteins secreted after stem cell therapy may promote regeneration in a paracrine effect which is related to the inherent cardiac stem cells [9]. These cells have only recently been introduced into the field and there are still several obstacles to overcome prior to advancing his platform to the clinic. Cardiac stem cells have been difficult to isolate and to expand ex vivo into meaningful numbers without losing differentiation potential and several groups are exploring ways to improve this cell population [10].

Bone marrow derived stem cells (BMSCs) have been utilized in variety of indications within the heart. These cells have primarily been to assist in the angiogenesis process and to assist in the revascularization of hibernating tissue. Typical clinical applications have been in acute MI and myocardial ischemia. Pompilio et al. (Arteriocyte, Inc.) demonstrated long term clinical and perfusion improvements in the absence of adverse events when injecting bone marrow derived stem cells into patients with myocardial ischemia [11].

Adipose tissue consists of adipocytes and a mononuclear cell fraction that contains a mesenchymal stem cell population. These cells are very similar in nature to bone marrow derived stem cells and in some cases have advantages over bone marrow derived stem cells. In one study by Zhang et al., adipose derived stem cells (ADSCs) exhibited a higher percentage of differentiating into cardiomyocytes when compared to bone marrow mesenchymal stem cells (MSCs). These results along with an advantage in tissue content, homology, growth and differentiation rate indicate that ADSC may be better suited for cellular cardiompoplasty than MSCs [4].
There has been some research in the use of peripheral blood and collection of stem cells for use in cardiovascular diseases. Baxter has recently completed a phase II trial of 150 patients to assess the safety and efficacy of CD34+ cells in patients with chronic myocardial ischemia. Patients receive granulocyte colony stimulating factor to mobilize the hematopoietic (blood-forming) CD34+ cells from their bone marrow to their bloodstream. Then, a cell separation system collects a mononuclear cell preparation rich in CD34+ stem cells from the patient's bloodstream and separates the cells using magnetic for use in the heart [12].

Skeletal myoblasts have been studied in over 2000 animals, 350 patients and was one of the first stem cells to enter into the clinical for cardiovascular diseases. A variety of groups have studied this platform with a variety of trial designs. The human body cannot, without medical assistance, repopulate regions of scar tissue within the heart with functioning muscle. Unlike other cells that have the capacity to fuse with other myoblasts are designed to improve cardiac function by populating regions of scar tissue. Myoblasts, which are obtained from a biopsy of the thigh, are precursors to muscle cells that have the capacity to fuse with other my oblasts or with damaged muscle fibers to regenerate skeletal muscle. When injected into scar tissue within the heart wall myoblasts have been shown to express various proteins that are important components of contractile function. The use of myoblasts obtained from a patient's own body helps to avoid certain challenges currently faced by other cell-based clinical therapies intended to be used for the treatment of chronic heart damage including tissue rejection and instances of the cells differentiating into cells other than muscle [4]

Embryonic stem cells may also have a potential role in cell therapy. Currently, however, these cells are available only in limited numbers, and their therapeutic use would likely introduce ethical and regulatory dilemmas as well as the risk of allograft immunologic reactions. Until now, it has not been possible to promote, in an in vitro culture, the development of layer of pure cardiomyocyte lineages derived from embryonic cells [7].

Though stem cells of various origins that show plasticity to cardiomyoctes are identified, this paper focuses more on study trial principally on BMSCs as prototypes. In a series of reports, it has been suggested that adult BMSCs has been established by identifying specific cell surface markers or by fluorescence in situ hybridization identification of Y-positive nuclei in donor-derived cells that have acquired the capacity to synthesize specific protein in regenerating tissues.

\section{Stem Cells Repopulation and Mechanisms of Repair}

\section{Technique for stem cell administration}

Administration of different types of progenitor cells by means of endovascular (intravenous or intracoronary) injection has been attempted in pilot studies, with promising early results. Readily available angioplasty catheters can be used for the intracoronary route but may entail a risk of microvascular obliteration and of poor therapeutic efficiency if the stem cells are to cross the coronary arteries. Bone marrow-derived stem cells appear to migrate through the arterial or capillary wall better than do skeletal myoblasts. The vascular approach seems generally less promising than direct intramural injection into the target myocardium, either surgically (via the epicardial approach) or by catheter via the endocardial approach [7].

Although the ideal route for administering stems cells is still yet to be determined, it may be important to take certain factors into 
consideration. The strength of homing signals may vary in different clinical scenarios. In more acutely ischemic cardiomyopathies, injection of the cells directly into the cardiac muscle may produce a more favorable outcome. Certain stem cells, such as skeletal myoblasts, are best administered by means of direct tissue injection because of the potential for demobilization when large numbers of these cells are administered [5].

\section{Mechanisms of repair}

Current evidence favors the conclusion that embryonic stem cells have multiple possible pathways of differentiation and that the embryonic interstitial matrix normally carries the messengers and inductors of final choice (which are not yet well known). Experimental embryology data have recently shown that extracardiac mesoderm does not normally produce heart tissue but can do so when transplanted into a specific cardiac extracellular medium. Likewise, extracardiac posterior mesoderm does not normally produce heart tissue but will express a cardiac phenotype when treated with extracellular regulators such as BMPs, FGFs, Wnt, and Dickkopf-1. This phenomenon suggests that the embryonic cardiac jelly, on extracellular matrix, is a critical carrier of signaling instructions and enables the cellular migration necessary for the acquisition of proper intercellular spatial and functional organization. For the formation of definitive cardiac chambers, myocardial specialization (into atria, ventricular, and conduction elements) is required. This necessitates the influence of several genes, such as those that encode peptide atria natriuretic factor; Hand 1 , cited 1 , and Irx 1/2/3; gap junction protein cannikins 40 and 43; the secreted peptide atria natriuretic factor; and the cytoskeleton protein Chisel [7].

It has been shown that stem cell homing molecules Stroma-cell Derived factor-1 (SDF-1) is transiently expressed following MI, and that re-establishment of this homing factors in myocardial tissue months after MI is sufficient to induce stem cell homing, vasculogenesis and recovery of myocardial function. SDF-1 is a family of CXC chemokines, and its receptor is CXCR4. The SDF-1/CXCR4 pathway is critical during embryogenesis for hematopoietic, vascular development, and cardiac development. It has been reported that 1) cells expressing markers of hematopoietic stem cells or EPCs express CXCR4; 2) vascular endothelial growth factor (VEDF) induces the expression of VEGF and induces angiogenesis in vivo [13-17]. SDF-1 and its receptor CXCR4 are crucial for bone marrow retention of hematopoietic stem cells and are involved in cardiogenesis and recruitment of endothelial progenitor cells to sites of ischemic tissue [18].

The mechanisms by which BMC therapy may improve cardiac function are still debated and not entirely clear. Whereas initial experimental studies had suggested a rapid transdifferentiation of BMCs (c-kitp, lineage-) into cardomtocytes after cardiac injection post-MI, probably inspired by the concept of a high stem cell plasticity of adult stem cells, later experimental studies using genetic techniques to follow bone marrow cell fate reported that transdifferentiation of BMCs into cardiomyocytes did not explain the observed effects of BMCs on LV function, and suggested that BMCs act rather by paracrine mechanisms to improve cardiac function, such as by stimulation of capillary growth, prevention of cardiomyocyte apoptosis or stimulation of resident cardiac stem cells. In fact, inhibition of endogenous mobilization of stem cells from bone marrow after experimental MI augmented myocardial damage after $\mathrm{MI}$ and resulted in an impaired capillary growth in the infarct border zone [19-21].

\section{In vivo tracking of transplanted stem cells}

Several recent meta-analyses of most controlled clinical studies have suggested a moderate, but significant, improvement of LVEF by MBC therapy in patients after MI. However, it should be noted that several of other established clinical therapies with an impact on prognosis in patients with $\mathrm{MI}$ and a reduced LV function, such as angiotesin converting enzyme (ACE) inhibitor or beta $(\beta)$-blocker therapy, are associated with similar improvement in LVEF and have been observed in patients after MI with a less optimal background therapy as compared with studies with BMC therapy. There are however, many remaining open question with respect to mechanism of stem/ progenitor cell therapy after MI and potential strategies to optimize its effects [21]. Moreover, several researches conducted so far to evaluate the efficacy of stem cell therapy for MI relied up on improvement in the LVEF. However, the presumptive increase in the LVEF would be obscured by numerous mechanistic artifacts. Therefore, tracking the fate of implanted stem cells in to the heart using imaging technology would give a relatively reliable response of stem cell therapy after MI.

Imaging will play an important role in assessing the myocardial response to stem cell therapy. In preclinical trials, analysis of tissue specimens allow detailed cellular characterization of genetic and cell surface markers. However, the need to euthanize the animal at fixed time intervals raises the complementary need for serial noninvasive imaging of myocardial function, perfusion, viability, and cell tracking [19]. Ideally, imaging technology used for stem cell tracking could have single-cell sensitivity is especially important in a new field such as that of stem cells because the pattern of migration of stem cells, even after local injection, is unknown and, there is a distinct possibility that single stem cells scattered diffusely through the body might be effective therapeutics for certain disease states. Moreover, the ideal imaging technology would permit tracking of injected stem cells for months to year because clinical trials undoubtedly will require long-term followup of tissue function or host survival [20].

Echocardiography assessment of myocardial function is well established. Doppler tissue imaging now provides real-time quantitative measurements of regional contractile function that have higher temporal resolution than any measurement scheme other than implanted ultrasonic crystal. Similarly, single photon emission computed tomography (SPECT) and positron emission tomography (PET) imaging can determine myocardial perfusion and viability. Developments in cardiovascular MRI warrant further discussion because this technology is well suited to serial studies. MRI is generally accepted as a gold standard method for evaluating cardiac anatomy and volumes. Real time visualization now allows identification of regional anatomy and volumes. Recent developments have, however, taken MRI beyond traditional anatomic and functional imaging. Realtime visualization now allows identification of regions of myocardial infarction and precise MRI-guided delivery of therapeutic agents. Furthermore, the injections sites can be identified using contrast agents. It is also feasible to label stem cells is with iron particles and detect their distribution in the body using MRI non-invasively. Noninvasive imaging can monitor the response to stem cell therapy at approximately the level of gross pathology. There is still a need to develop techniques that can detect the cells introduced into the myocardium and to follow their division over time. There are important subtleties of cellular function, growth, and proliferation that cannot be imaged with the kinds of noninvasive methods described. Thus, there will remain substantial need for detailed physiological and pathological methods to understand the myocardial response to stem cell therapy [19].

\section{Morbid state and homing}

The homing of BM-progenitor cells to the damaged myocardium, 
which results in cell engraftment, may play a key role in the success of cell therapy. After acute ischemic myocardial injury, serum stoma cell derived factor- 1 levels rise significantly, and this chemokine appears to be one key homing signal that regulates homing of stem and progenitor cells to the ischemic myocardium. In patients with chronic ischemic cardiomyopathy, however, local homing signals may not be as intense as in the early post-infarction phase, and therefore the coronary route might not be optimal for cell engraftment [13].

Bone marrow derived stem cells work best in an acute setting or in hibernating tissue mainly due to the fundamental chacteristiics of mesenchymal stem cells. Bone marrow derived mesenchymal stem cells are medium-dependent and tend to acquire the characteristics of the cells they are in contact with. For this reason, these cells are not appropriate for the scar tissue of a chronic myocardial infarct (i.e., into the fibrotic area, because they would tend toward differentiating into more fibrosis) [16].

Cytori therapeutics has recently completed enrollment in study to investigate adipose derived stem cells in chronic heart disease. The phase I PRECISE trial was carried out at several centers in Europe with enrollment of 27 patients. The independent data safety and monitoring board had not identified any safety concerns and six month results are expected in the first half of 2010 [14]. Cytori is also currently recruiting for a phase I study at two centers in Europe to establish the safety and feasibility of Adipose derived stem cells in patients with AMI [15].

\section{Constraints and Options}

\section{Ethical issues}

Though embryonic stem cells have also a potential role in cell therapy, these cells currently are available only in limited numbers, and their therapeutic use would likely introduce ethical and regulatory dilemmas as well as the risk of allograft immunologic reactions [7].

\section{Biology of senescence}

Myocardial infarction is primarily a disease of older persons, and the senescent myocardium may differ biologically from the myocardium of young persons and from that of the small adult animals typically used in initial stem cell experiments. In particular, the cells (myocytes) them self and the intercellular messaging milieu in the interstitial space may be profoundly different under each clinical condition. Nadal-Ginard et al. [22] recently characterized the senescent myocardium by the predominance of large myofibres (volume $>90,000$ $\mathrm{mm}^{3}$ ) expressing p16INK4, a marker of cellular aging and increased apoptosis. Most likely, the molecular signals produced by such cells and their extracellular environment are not as favorable for stem cell differentiation, migration, and integration as are the signals present in younger hearts. These conditions need to be further characterized, because they might allow physicians to modify the environment, making it more conducive to successful stem cell treatment [7].

\section{Miscellaneous factors}

In contrast to oral medication pharmacological agents, BMC therapy is an invasive treatment option. Bone marrow aspiration is usually performed under local anesthesia and is generally well tolerated. However, the procedure is without doubt a strain for the AMI patient and stent thrombosis has been described. Alternatively, the aspiration procedure can be performed under a brief general anesthesia. Both this procedure and the repeated left-sided heart catheterization for cell administration expose the patient to the risk of possible procedurerelated complications. Coronary artery dissection has been reported after BMC administration. Furthermore, there has been concern about increased rate of restenosis after BMC therapy, although this has not been reported after injection of unfractioned cells in the absence of bone marrow mobilizing agents. Another aspect s that MBC therapy is unlikely to transfer to a clinically important improvement in patient outcomes and that it probably not outweighs the cost, effort, and risks of a large-scale clinical trial [21].

Despite several limitations, there are alternative ways to acknowledge the potential of BMC therapy. Considering the well-known limitations of the methods that have been used, it is amazing that there seems to be any effect of this treatment. First, we have not identified the cell (s) with a possible regenerative effect. Second, we do not know the mechanism of action. Third, cell numbers that have been delivered are probably too low to expect clinically relevant myocardial regeneration [23]. Fourth, few cells remain in the heart regardless of administration mode [24], and finally, most of the few cells that remain in the heart die shortly after delivery [25] of AMI patients. Further research will probably develop techniques that will overcome these obstacles. It is possible to deduce that, the field is not yet ready for large-scale trial, and it will be better to use resources on more basic research and continue small to intermediate sized, well-designed clinical trials.

\section{Conclusion}

While the initially perceived rapid chance for a complete cardiac repair by stem/progenitor cell therapy after MI has generated high expectations, now the potential of this therapy needs to be carefully developed by addressing important remaining questions, including the optimal cell types and preconditioning, the timing and dosing cells to be seed, how to augment the functional repair capacity of transplanted cells, how to optimize their homing and engraftment in the heart, and how to select the patients that may benefit most from this therapy.

\section{Conflict of Interests}

The authors declare that they have no competing interests.

\section{Acknowledgements}

The authors are grateful to Ambo University for provision internet services.

\section{References}

1. British Heart Foundation (BHF): Coronary heart disease statistics: fact sheet.

2. Roger VL, Go AS, Lloyd-Jones DM, Adams RJ, Berry JD, et al. (2011) Heart disease and stroke statistics--2011 update: a report from the American Heart Association. Circulation 123: e18-e209. [PubMed]

3. Lloyd-Jones DM, Hong Y, Labarthe D, Mozaffarian D, Appel LJ, et al. (2010) Defining and setting national goals for cardiovascular health promotion and disease reduction: the American Heart Association's strategic Impact Goal through 2020 and beyond. Circulation 121: 586-613. [PubMed]

4. Comella K, Griffeth J (2009) Stem Cell Research in the Cardiac Field: Where Are We Now? World Stem Cell Report.

5. Perin EC, Geng YJ, Willerson JT (2003) Adult stem cell therapy in perspectives. Circulation 107: 935-938. [PubMed]

6. Forester JS, Pric MJ, Makkar RR (2003) Stem cell repair of infarcted myocardium: An overview for clinicians. Circulation 108: 1139-1145. [PubMed]

7. Angelini P, Markwald RR (2005) Stem cell treatment of the Heart: Are view of its current status on the Brink of clinical Experimentation. Tex Heart Inst J 32: 479-488.

8. Beltrami AP, Barlucchi L, Torella D, Baker M, Limana F, et al. (2003) Adult cardiac stem cells are multipotent and support myocardial regeneration. Cell 114: 763-776. [PubMed]

9. Stastna M, Abraham MR, Van Eky JE (2009) Cardiac stem /progenitor cells secreted proteins, and proteomics. FEBS lettes 583: 1800-1807. [PubMed] 
Citation: Satessa GD, Lenjisa JL, Gebremariam ET, Woldu MA (2015) Stem Cell Therapy for Myocardial Infarction: Challenges and Prospects. J Stem Cell Res Ther 5: 270. doi:10.4172/2157-7633.1000270

Page 5 of 5

10. Barile L, Messina E, Giacomello A, Marian E (2007) Endogenous cardiac stem cells. Prog Cardiovasc Dis 50: 31-48. [PubMed]

11. Pompilio G, Teinoff G, Liebold A, Pesce M, Alamanni F, et al. (2008) Direct minimally invasive intramyocardial injection of bone marrow-derived AC133+ stem cell in patients with refractory ischemia: preliminary results. Thorac Cardiovasc Surg 56: 7-6. [PubMed]

12. http://www.clinicaltrials.gov/ct/show/NCT00300053?order $=1$

13. Goussetis E, Manginas A, Koutelou M, Pristri I, Teodosaki M, et al. (2006) Intracoronary infusion of CD 133 and CD133 CD34 selected Autonomous bone marrow Progenitor cells in patients with chronic ischemic cardiomyopathy: cell isolation adherence to the infracted area, and body distribution. Stem Cells 24 2279-2283.

14. http://ir.cytoritx.com/releasedetail.cfm?ReleaselD=386027

15. http://www.clinicaltrials.gov/ct2/show/NCT0044280?term=cytori\&rank=1

16. Souza LC, Carvalho KA, Rebelatto C, Senegalia A, Furuta M, et al. (2004) Combined transplantation of skeletal myoblast and mesenchymal cells (cocultivation) in ventricular dysfunction after myocardial infarction. Arg Bras Cardiol 83: 294-299. [PubMed]

17. Hiasa K, Ishibashira K, Inoue S, Zhao Q, Kitamoto S, et al. (2004) Gene transfer of stromal cell-derived factor-1 enhances ishemic vasculogenesis and angiogenesis via vascular endothelial growth factor/Endothelial Nitric Oxide syntheses-related path way. Circulation 109: 2454 -2461.
18. Askari MD, Unzek S, Corey GK, Ellis SG, Thomas JD, et al. (2004) Cellular, but not direct, adenoviral delivery of vascular endothelial growth factor result in improved left ventricular function and neovascularization in dilated ischemic cardiomopathy. J Am Coll Cardiol 43: 1908-1914. [PubMed]

19. Orlic D, Hill JM, Arai AE (2002) Stem cells for myocardial regeneration. Circ Res 91: 1092-1102. [PubMed]

20. Frangioni JV, Hajar RJ (2004) In vivo tracking of stem cells for clinical trials in cardiovascular diseases. Circulation 110: 3378-3383. [PubMed]

21. Ulf $L$ (2009) Bone marrow cell therapy after myocardial infarction: What should we select? Eur Heart J 30: 1310-1312. [PubMed]

22. Nadal-Ginard B, Kajstura J, Leri A, Anersa P (2003) Myocyte death, growth, and regeneration in cardiac phypertrophy and failure. Circ Res 92: 139-150. [PubMed]

23. Nadal-Ginard B, Faster V (2007) Myocardial cell therapy at the crossroads. Nat Clin Pract Cardiovasc Med 4: 1. [PubMed]

24. Hou D, Youssef EA, Brinton TJ, Zhang P, Rogers P, et al. (2005) Radiolabeled cell distribution after in tramyocardial, intracoronary, and interstitial retrograde coronary venous delivery: Implications for current clinical trials. Circulation 112 150-156. [PubMed]

25. Muller-Ehmen J, Krausgrill B, Burst V, Schenk K, Neisen UC, et al. (2006 Effective engraftment but poor mid-term persistence of mononuclear and mesenchymal bone marrow cells in acute and chronic rat myocardial infarction J Mol Cell Cardiol 41: 887-884. [PubMed] 